\title{
Physiological basis of stretching study in the training system of young football players
}

\author{
Natalya Yu. Tarabrina*, Yuriy V. Kraev \\ Moscow Aviation Institute (National Research University) \\ Moscow, Russia \\ ORCID: oooo-ooo3-1469-5010, nata-tarabrina@mail.ru* \\ yury.kraev@mail.ru
}

\begin{abstract}
For a successful game in football it is necessary to develop the main physical qualities and improve all functional systems of the athletes' organism. It is possible only owing to many-sided physical training, when together with the main physical qualities development attention is paid to football players' special qualities development. The article gives physiological substantiation of muscle relaxation role in the mechanism of young football-players' accuracy of movements formation. Materials. Myotonic and coordinating indices are studied among 22 young athletes, who are 8-12 years old. All respondents trained according to specially created three-week program. It included the exercises for paravertebral muscles and lower extremities muscles stretching. Methods. Information sources analysis and summarizing, electromyotonometry, pedagogical experiment, methods of mathematical statistics. Results. It is shown that stretching exercises fulfillment decreases myotonus among all the respondents in the following points: V37, E 32, V52, V 57 (sinister и dexter) from 3,52\% till 9,76\% (p<0,05-0,001).The most susceptible to such kind of influence turned out to be calf muscles and a front surface of the hip, less susceptible turned out to be lumbar part of the muscle, which straightens the spine. Myogenic tonus decrease leads to the quality of movements improvement accuracy from 25,0 till 37,05\% (p<0,05). 11-12-year-old football players had the same myovisceral reactions as 8-9-year-old athletes, however, the degree of these reactions intensity was more considerable. Conclusion. The expediency of stretching use was considered. We proved the necessity to use this methodology during transfer and preparatory period of sports training among young football players.
\end{abstract}

Keywords: young football-players, stretching, myogenic tonus, purpose-oriented accuracy of movements.

For citation: Natalya Yu. Tarabrina*, Yuriy V. Kraev.Psychological basis of stretching study in the training system of young football players. The Russian Journal of Physical Education and Sport. 2018; 13(4): 134140. DOI 10.14526/2070-4798-2018-13-4-161-169

\section{Introduction}

For a successful game in football it is necessary to develop the main physical qualities and improve all functional systems of the athletes' organism. It is possible only owing to many-sided physical training, when together with the main physical qualities development attention is paid to football players' special qualities development [1].

It is known that the most important qualities of a football player are the following: explosive speed and movements coordination. Endurance and strength should only support these qualities [1,2]. At the same time, speed and coordination are connected, as speed of difficult in coordination movements fulfillment very often happens in terms of severe time deficiency. It is important to connect speed and accuracy, which depends on eurhythmy of intramuscular and intermuscular coordination processes.

Different forms and character of accuracy (purpose-oriented accuracy of movements (PAM)), connected with different physiological systems of an organism, demands special approach to its development.

According to some research works (Asshauera and others) the use of exercises with muscles and tendons tension and stretching alternation has a positive influence on movements coordination [3]. This method of exercises fulfillment is called stretching. In the opinion of Solverborn [6] the essence of the method is "in the definite muscles tension and relaxation and some strain alternation". It means that 
such kind of exercises lead to the state of muscles tension-relaxation and strain and it provides the main techniques fulfillment - kicks, sliding tackles, feints with maximum amplitude. It should be noted that good flexibility proves the fact that muscles of a football player are flexible plastic and are in a good state.

However, there is no one methodical approach to the mechanism of stretching influence on athletes. Some authors think that stretching generally influences nervous-muscular apparatus, improves stimulation transfer from the nerve to muscle fibre [3]. During muscle stimulation nervous-muscular excitation transfer intensification is caused by the increase of acetylcholine release from nerve endings. It leads to the amplitude of end plates potentials increase. New fibres involvement into activity is partially provided by their excitatory tendency increase. Other scientists think that stretching directly influences muscle fiber and changes its dynamic characteristics $[2,4]$. Some scientists proved that stretching in terms of an integral organism is a specific proprioceptors stimulus and influences central nervous system with further reflex influence on neuromuscular apparatus [5].

Swedish school offers both traditional gymnastic exercises, which influence muscles flexibility increase and bursts, which prevent reflex contraction (stretch muscle fibres) [3].

American school of stretching popularizes body-building exercises, which are based on muscle fibres stimulation. We speak about extension and strain of muscles-antagonist (6 seconds each fulfillment during one minute) [3].

Some specialists, in particular Solverborn, characterize stretching as the system of exercises, during which the ability to stretch is developed on the basis of the following principle: muscles tension-relaxationstretching and the state of strain preservation. It leads to joints flexibility improvement [6].

V.A. Levtov and other authors (1985) revealed that "stretching or contraction influence not only muscles proprioceptos, but also directly muscle fibres. Moreover, violent stretching causes tension in a muscle approximately $1 / 5$ more than isometric contraction, caused by electric stimulation of a muscle. Useful activity fulfilled directly after stretching can be bigger, than the work of the same muscle during contraction in the same conditions, but from isometric contraction state, it means without stretching. It is connected both with greater contraction ability of a muscle, developed after stretching, and flexible energy accumulation".

The aim of the research - to study the role of muscle relaxation in the mechanism of purposeoriented accuracy of movements formation among young football players.

\section{Materials and Methods}

22 young 8-12-year-old football players, who train in football club "TSK-Tavriya" in Simferopol, took part in the research. All respondents were divided into two groups: the first group included ten 8-10-year-old people. The second group included twelve 11-12-year-old people, who also go in for football. All respondents had the third junior category. During 21 days at the end and the beginning of each training the respondents from both groups fulfilled a complex of physical exercises during 18-20 minutes - stretching, created by Swedish sports specialist Extrand [3,7,9] and which included exercises for paravertebral muscles and lower extremities muscles stretching. The methodical complex is realized in the following way: during 10 minutes players move with the ball using low intensity. Then during 5 minutes the exercises are fulfilled for hip and knee joints putting and abduction. It means muscles tension and stretching in the following order: 8-10 seconds - tension, 2-3 seconds - relaxation, 8-10 seconds - stretching. After this complex fulfillment players fulfill the exercises with the ball (fulfilling them 5-8 minutes more) with maximum efforts. Then stretching exercises are repeated with bigger amplitude and the time increase of tension preservation in muscles till 10 seconds. The complex includes the following stages:

The first week: tension - 18-20 seconds, relaxation - 2-3 seconds, stretching - 8-12 seconds (using own body weight).

The second week: tension -1 -25 seconds, relaxation - 7-12 seconds, stretching -1 -15-25 seconds 
(using the barriers, benches, wall-bars and others).

The third week: tension - 25-30 seconds, tension - 10-15 seconds, stretching - 25-30 seconds (with the help of a partner, using sports equipment, with not heavy poundage).

All athletes didn't have training lessons one day before the survey and on the day of survey.

Before and after three weeks of trainings muscles tonus of lower extremities and lumbar spine estimation was held with the help of "NOVOTEST" (Russia) electricmyotonometer, created according to S.F. Baronov and Yu.M. Uflyanda system, for the lying position of the respondent. Myotonometric research was held in symmetric biologically active points: V - 37 "In-men", situated in the middle of the back hip surface, E - 32 "Fu-tu" on anterolateral surface of the hip, V - 52 "Chzhi-shi", situated in the gap between the $2^{\text {nd }}$ and the $3^{\text {rd }}$ acanthi of lumbar vertebrae, $\mathrm{V}-57$ "Chen-shan", situated in the center of the back hip surface, in the middle of the line, drawn from the upper edge of a heel bone to the center of popliteal space $[5,10]$.

We used "purpose-oriented accuracy of movements determination" test, created by S.V. Golomazov 1996 (PAM), for the control over muscular efforts dosing and the set spatial characteristics of movements reproduction $[1,8,11]$.

Calculations and graphic design of the received in work results was held with the help of Microsoft Excel program and "STATISTICA - 10.0" software package. The criterion selection for statistical hypotheses and central tendencies measures, which describe the results, was held depending on the results of distribution check. The distribution was held with the help of Shapiro-Wilk test. Then, in case of accepting an alternative statistic hypothesis about the differences of the feature, a pair comparison of groups was held using nonparametric Wilcoxon signed-ranks test. In this case as the measure of central tendency median was used (Me), as the measure of dispersion- the lower quartile (Q1) and the upper quartile (Q3) (25 and 75 percentiles). In order to check null hypothesis about differences absence of the connected groups with normally distributed feature, at the beginning we used the procedures of single-factor analysis of variance of the repeated measures (Repeated Measures ANOVA). Then normality of difference distribution between the feature values for pair Student criterion and the condition of sphericity for repeated measures variance analysis. Then, in case of accepting an alternative statistic hypothesis, a pair comparison of groups was held using parametric Student's test. In this case as central tendency measure the arithmetical mean (M) was used, as measure of dispersion we used a standard error of the arithmetical mean (m).

\section{Results and Discussion}

During the effects of stretching study among young football players we revealed high level of the studied indices sensitivity to this factor. The results of the held research works prove that in intact state muscle tone of the respondents from both groups didn't differ validly.

After lessons with stretching in the first group muscle tone decreased validly in 5 points from 8 studied points. The most significant change was in $\mathrm{V} 37$ sinister $-9,37 \%(\mathrm{p}<0,001)$ point, the least significant changes was in $\mathrm{V}_{5} 2$ sinister - 2,00\% (table 1).

It was stated that the athletes of the second group had a valid myotonus decrease in all studied symmetrical point after stretching: V 37 and E 32 - till 10,2\% (p<0,001) and 8,01\% (p<0, 001), in V52 and V57 points - till 11,76\% ( $\mathrm{p}<0,001)$ and $8,87 \%(\mathrm{p}<0,001)$ (table 1; picture 1).

Comparative analysis of relative values changes of myotonus indices in the first and the second groups helped to reveal the differences in all points ( $\mathrm{p}<0,05^{-0,001)}$, except VG37 sinister point. The difference in VG37 dexter point was 6,12\%, in E32 sinister point - 4,62\%, in E32dexter point- 4,01\%, in V52 sinister point - 9,76\%, in $\mathrm{V}_{5} 2$ dexter point - 3,52\%, in V57 sinister point - 6,25\%, in V57 dexter point$4,84 \%$ (picture 1). Stretching effect for myotonus decrease is was more vivid in the group of 11-12-year-old athletes. 
Table 1 - Muscle tone change in segments L3- S2 and lower extremities among young football players $(n=22)$ before and after the cycle of training using stretching

\begin{tabular}{|c|c|c|c|c|c|c|}
\hline \multirow{2}{*}{$\begin{array}{c}\text { Points, unit of } \\
\text { measure }\end{array}$} & \multicolumn{3}{|c|}{ The first group $(n=10)$} & \multicolumn{3}{|c|}{ The second group $(n=12)$} \\
\hline & Before & After & $\Delta, \%$ & Before & After & $\Delta, \%$ \\
\hline $\begin{array}{l}\text { V } 37 \text { sinister, } \\
\mathrm{m}\end{array}$ & $\begin{array}{c}23,33 \pm \\
1,91\end{array}$ & $\begin{array}{c}21,33 \pm \\
1,84\end{array}$ & $\begin{array}{c}-9,37 \\
* * *\end{array}$ & $\begin{array}{c}21,0 \\
(20,0-23,0)\end{array}$ & $\begin{array}{c}19,0 \\
(18,0-20,0)\end{array}$ & $\begin{array}{l}-9,52 \\
\wedge \downarrow\end{array}$ \\
\hline V 37 dexter, $\mathrm{m}$ & $\begin{array}{c}51,0 \\
(49,0-52,0)\end{array}$ & $\begin{array}{c}49,0 \\
(47,0-50,0)\end{array}$ & $\begin{array}{c}-4,08 \\
\wedge\end{array}$ & $\begin{array}{c}49,0 \\
(47,0-51,0)\end{array}$ & $\begin{array}{c}44,0 \\
(42,5-46,0)\end{array}$ & $\begin{array}{c}-10,20 \\
\downarrow\end{array}$ \\
\hline $\begin{array}{c}\text { E } 32 \text { sinister, } \\
\mathrm{m}\end{array}$ & $\begin{array}{c}50,25 \pm \\
2,08 \\
\end{array}$ & $\begin{array}{c}48,39 \pm \\
2,61 \\
\end{array}$ & $\begin{array}{c}-3,85 \\
* * * \\
\end{array}$ & $\begin{array}{c}48,56 \pm \\
2,79 \\
\end{array}$ & $\begin{array}{c}44,44 \pm \\
2,89 \\
\end{array}$ & $\begin{array}{c}-8,47 \\
* * * \\
\end{array}$ \\
\hline E 32 dexter, $\mathrm{m}$ & $\begin{array}{c}52,0 \\
(50-53)\end{array}$ & $\begin{array}{c}50,0 \\
(49,0-52,0)\end{array}$ & $\begin{array}{c}-4,00 \\
\downarrow\end{array}$ & $\begin{array}{c}50,28 \pm \\
2,05 \\
\end{array}$ & $\begin{array}{c}46,25 \pm \\
3,26 \\
\end{array}$ & $\begin{array}{c}-8,01 \\
* * *\end{array}$ \\
\hline V52 sinister, $\mathrm{m}$ & $\begin{array}{c}51,0 \\
(50,0-52,5)\end{array}$ & $\begin{array}{c}50,0 \\
(48,0-52,0)\end{array}$ & $\begin{array}{c}-2,00 \\
\downarrow\end{array}$ & $\begin{array}{c}51,0 \\
(49,0-52,0)\end{array}$ & $\begin{array}{c}45,0 \\
(44,5-47,5)\end{array}$ & $\begin{array}{c}-11,76 \\
\downarrow\end{array}$ \\
\hline V52 dexter, $\mathrm{m}$ & $\begin{array}{c}41,96 \pm \\
1,61\end{array}$ & $\begin{array}{c}39,83 \pm \\
2,29 \\
\end{array}$ & $-5,35$ & $\begin{array}{c}40,70 \pm \\
2,64 \\
\end{array}$ & $\begin{array}{c}37,09 \pm \\
2,33\end{array}$ & $\begin{array}{c}-8,87 \\
* * *\end{array}$ \\
\hline V57 sinister, $\mathrm{m}$ & $\begin{array}{c}41,61 \pm \\
1,99\end{array}$ & $\begin{array}{c}39,83 \pm \\
2,95\end{array}$ & $-4,48$ & $\begin{array}{c}41,74 \pm \\
1,71\end{array}$ & $\begin{array}{c}37,26 \pm \\
1,74\end{array}$ & $\begin{array}{c}-10,73 \\
* * *\end{array}$ \\
\hline V57 dexter, m & $\begin{array}{c}41,78 \pm \\
2,65 \\
\end{array}$ & $\begin{array}{c}40,35 \pm \\
2,53 \\
\end{array}$ & $-3,56$ & $\begin{array}{c}40,87 \pm \\
2,58 \\
\end{array}$ & $\begin{array}{c}37,43 \pm \\
1,95 \\
\end{array}$ & $\begin{array}{c}-8,40 \\
* * * \\
\end{array}$ \\
\hline
\end{tabular}

Notes: here and further: ******* - validity of differences p<0,05, o, 01, o, 001, Student's test; $\downarrow \downarrow \downarrow, \downarrow \downarrow-$ validity of differences $\mathrm{p}<0,05,0,01,0,001$, Wilcoxon rank sum test, \# - tendency to the feature increase /decrease ( $<<0,1), \Delta \%$ - indices difference in $\%$

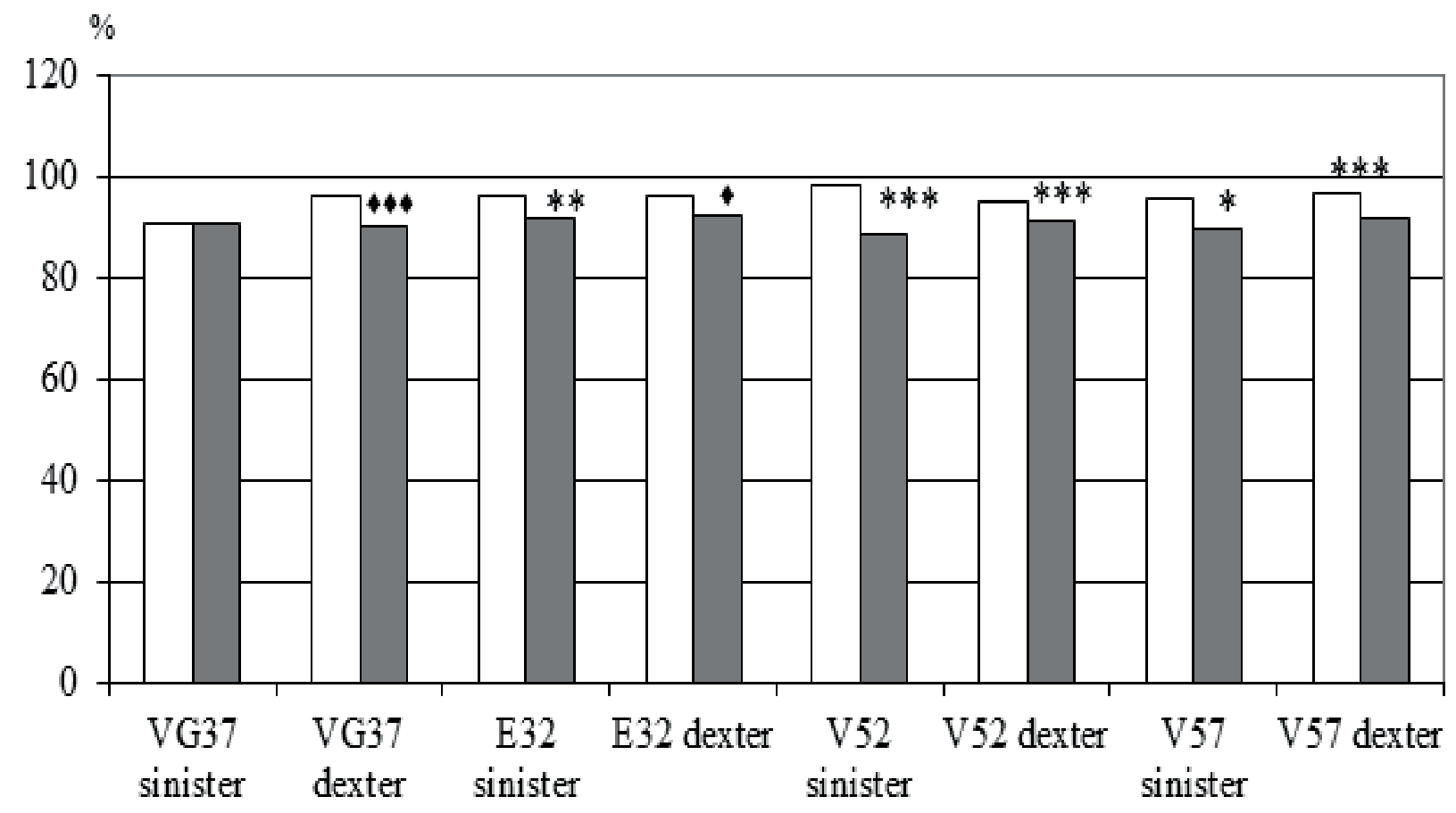

\section{ОI группа ОII групाа}

Picture 1 - The differences of relative values of myotonus (\%) among the athletes from the first $(n=10)$ and the second $(n=12)$ groups in terms of cycle of trainings using stretching in comparison with the initial level, taken as $100 \%$

I группа- the $1^{\text {st }}$ group

II группа- the $2^{\text {nd }}$ group

The fulfilled research works prove that at the beginning of the experiment the initial PAM indices of the respondents were at the average and below the average development level. According to the norms, 
adopted in a typical educational-training program of sports training for sport schools for children and teenagers and sport schools for children and teen-agers of the Olympic reserve (created by Russian football union as control normatives), it corresponds with "satisfactory" mark and shows a low training level $[3,12,13]$. Such kind of development level of spatio-temporal and coordinating abilities is connected with the problems of educational-training process among children and teen-agers and the absence of an integral methodical approach to this question (this fact can be considered as not a negative factor). That is why it is necessary to search for this problem solution $[14,15,16]$.

After the lesson according to the program, using stretching PAM test indices improved validly in both groups (picture 2).

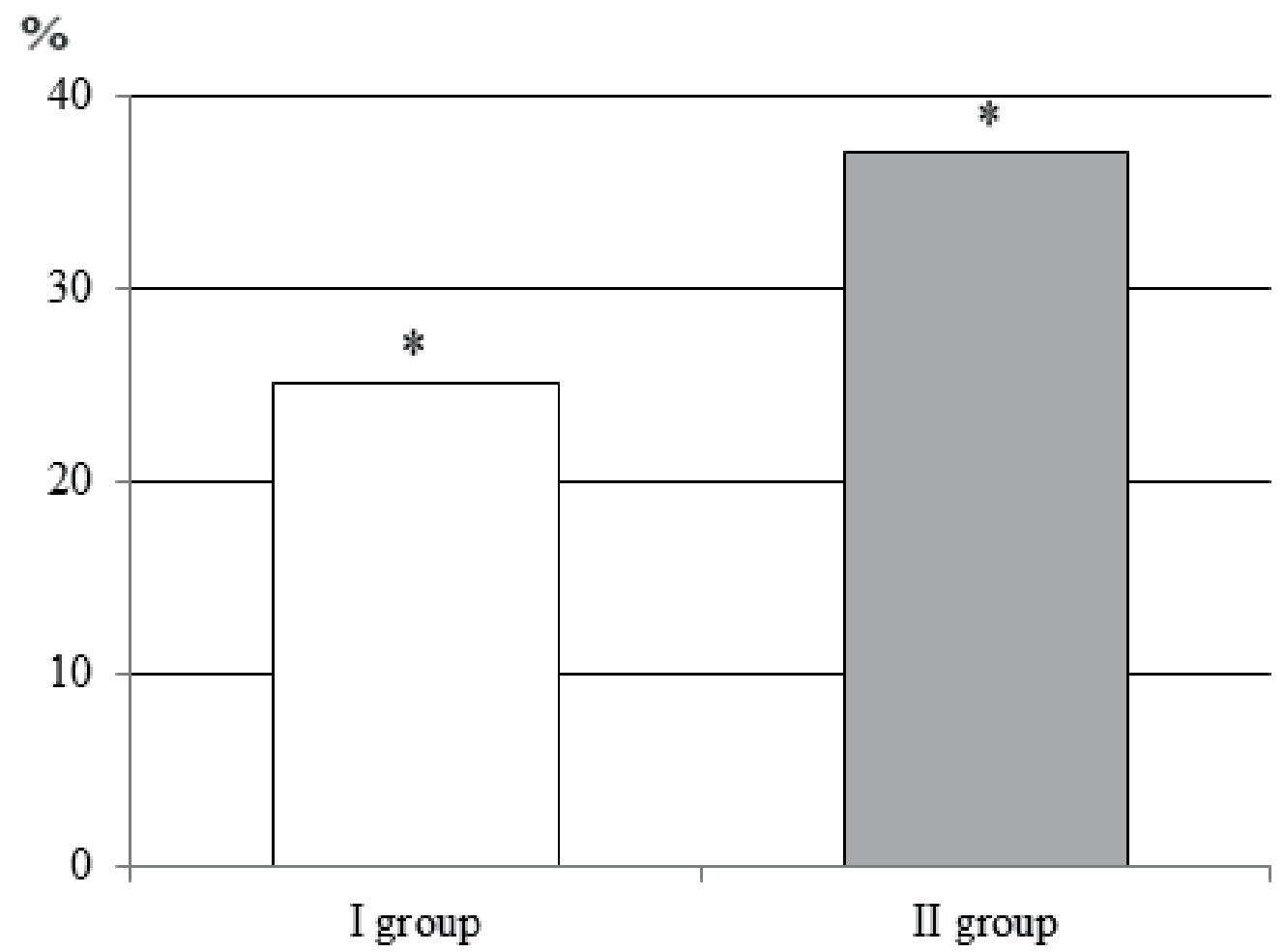

Picture 2 - The indices differences of coordination PAM test among young athletes football players $(\mathrm{n}=22)$ in terms of the training cycle with stretching (\%)

The results of the held research works prove that among 8-9 year-old children the cycle of stretching lessons provides the results of purpose-oriented accuracy of movements increase only by $25 \%(\mathrm{p}<0,05)$, increasing the number of hits from 4 to 5 out of 10 offered. Among 11-12 year-old children this index increased by $37,05 \%(p<0,05)$. The effectiveness changes from 3,40 $\pm 0,19$ c.u. to 5,00 $\pm 0,81$ c.u. out of 10 offered. It is obvious that the age-related dynamics of accuracy should provide the character of organism maturation for the corresponding skills mastering, which have different degree of coordinating difficulty. It is well seen in the degree of correlation change of different indices of motor qualities with the level of technical mastery. The received results correspond with the opinion of S.V. Golomazov and others, who say, that a considerable accuracy increase happens only in early age $-10-12$ years-old $[1,8,17]$. By this age a child's motor apparatus becomes mature and it is possible to fulfill such kind of tasks being on a par with adults. 11-12-year-old boys like masters are able to differentiate the separate parameters of the kick (speed or direction) [8]. It proves that accuracy of kicks is determined not by the mistakes in speed or direction, but in correct combination of these parameters selection, which is necessary for an accurate hit $[14,18]$.

In the opinion of S.V. Golomazov (2007) and others, "starting from the age of 11 during penalty shots in basketball there is equal variability according to speed and the angle of ball throw both among children and basketball experts" [1,8,17].

It should be noted that there is no unified methodical approach to the mechanism of stretching 
influence on PAM development [2-6,9].

The possible mechanism of skeletal muscles stretching influence on PAM is connected with proprioceptive sensitive ways, which connect nuclei of spinal cord, macromyelon, bridge, subcortex, extrapyramidal subsystem with cerebellum, which take part in the mechanisms of automatic movements coordination and muscles tonus, in addition to the ways, which are closed in brain cortex. As a rule these mechanisms are demonstrated during quick balance violations or automatic movements fulfillment, which are produced during special exercises fulfillment.

Unconditioned impulses from all mentioned above structures integrate in cerebellum, which coordinates and sets different in accuracy movements. Impulses from cerebellum have coordinating inhibitory influence on vestibular analyzer and reticular formation nuclei. As vestibular nuclei form vestibulospinal tract, there is oppression or facilitation of $\alpha$ - function and $\gamma$ - motoneurones of the front columns of the spinal cord and muscle spindles of motor peripheral nerves on it and on reticulospinal track. Thus, owing to the mechanisms of feedback through vestibulospinal and reticulospinal ways cerebellum coordinates quick and slow retractions of all muscles. Cerebellum is like a block of regulation, based on the principle of feedback. Vermis coordinates accuracy of movements. In cerebellum hemisphere there are mechanisms of purpose-oriented accuracy and coordination. Vermis is controlled by cerebellar cortex and it functions under the influence of cortex.

The results of present research work can be the base for further mechanisms study of coordinating influence of stretching skeletal muscles on somatic and visceral reactions of an organism in terms of different loads. They can be used for scientifically substantiated recommendations creation concerning stretching use in sports and rehabilitation practice.

\section{Conclusion}

1. Under the influence of the complex of stretching exercises there was myotonus decrease among all respondents in the following point: $V_{37}, \mathrm{E}_{32}$, V52, V 57, (sinister и dexter) from $3,52 \%$ to 9,76\% ( $\mathrm{p}<0,05-0,001$ ). The most sensitive to this influence turned out to be calf muscles and muscles of hip front surface, less sensitive to this influence turned out to be back part of the muscle, which straightens the spine.

2. Stretching use influences functional state of young football players' motor system and leads to spatio-temporal characteristics and the quality of accurate movements improvement from 25,0 to $37,05 \%(p<0,05)$. It can improve the effectiveness of competitive activity.

3. It is mentioned that among 10-12-year-old football players myovisceral reactions were the same as among 8-9-year-old athletes, however, the degree of these reactions' demonstration was more significant.

\section{References}

1. Tarabrina N. Yu. Influences of automyofascial release using a massage roller on the range of motion in the hip joint among athletes. Crimean journal of experimental and clinical medicine. 2018;8(1):75-83. [In Russ].

2. Solomonko V.V. Stretching in the training process of football players. Football- Profi. 2006;2(3):13-21. [In Russ].

3. Tarabrina N. Yu. Muscle relaxation in the system of scoliotic changes correction in athletes' cervical-thoracic spine. Theory and practice of physical culture. 2016; 4:30-33. [In Russ., In Engl.].

4. Patent 2603619 Russian Federation, IPC: A61H 33/oo A61H 39/oo A63B 31/10 Method of muscle-tonic asymmetry correction of a person's paravertebral zone / Tarabrina N.Yu., Grabovskaya E. Yu.; applicant and patentee V.I. Vernadsky Crimean Federal University, no 2015134116; Appl. 13.08.2015; publ. 13.11.2016. [In Russ].

5. S.M. Ostojic, M. Stojanovic, I. Jukic, E. Pasalic, M. Jourkesh. The effects of six weeks of training on physical fitness and performance in teenage and mature top-level soccer players. Biology of Sport. 2009; 26(4):379-387. [In Engl.]. 
6. Litvinov R. V. The mastery of football players' kicking actions assessment. Electronic scientific and methodical journal of Omsk State University. 2018;1 (12):36. [In Russ].

7. Tarabrina N. Yu. The peculiarities of specific fitness means influence on speed-power and coordinating indices of young football players. Science and sport: modern trends. 2016; 10.1 (10):31-35. [In Russ].

8. Shaykhelislamova F.G., Sitdikov M.V. Age - gender characteristics and mechanisms of adaptive reactions among children of pre-and pubertal periods of development. Human physiology. 2009.35;6:103110. [In Russ].

9. Ive Luz Canhadas, Rodrigo Lopes Pignataro Silva, Celso Rodrigures Chaves, Leslie Andrews Portes. Anthropometric and physical fitness characteristics of young male soccer players. Revista Brasileira de Cineantropometria e Desempenho Humano 2010; 12(4): 239-245.

10. Bradley P.S., Olsen P.D., Portas M.D. The effect of static, ballistatic and propreoceptive neuromuscular facilitation stretching on vertical jump performance. Journal of Strength and Conditioning Research. 2007; 21: 223-226.

11. Duthie G.M., Pyne D.B., Ross A.A., Livingstone S.G., Hooper S. The reliability of ten-meter sprint time using different starting techniques. Journal of Strength and Conditioning Research. 2006; 20: 246-251.

12. Fletcher I.M., Anness R. The acute effects of combined static and dynamic stretch protocols on fifty-meter sprint performance in track and field athletes. Journal of Strength and Conditioning Research. 2007; 21: 784-787.

13. Little T., Williams A.G. Specificity of acceleration, maximum speed and agility in professional soccer players. Journal of Strength and Conditioning Research. 2005; 19: 76-78.

14. Little T., Williams A.G. Effects of differential stretching protocols during warm-ups on highspeed motor capacities in professional soccer players. Journal of Strength and Conditioning Research. 2006; 20: 203-207.

15. McNeal J., Sands W. Acute static stretching reduces lower extremity power in trained children. Paediatric Exercise Sciences. 2003; 15: 139-145.

16. Pearce A.J., Kidgell D.J., Zois J., Carlson J.S. Effects of secondary warm up following stretching. European Journal of Applied Physiology. 2009; 105: 175-183.

17. Shrier I. Stretching before exercise does not reduce the risk of local muscle injury: A critical review of the clinical and basic science literature. Clinical Journal of Sport Medicine. 1999; 9: 221-227.

18. Young W.B., Clothier P., Otago L., Bruce L., Liddell D. Acute effects of static stretching on hip flexor and quadriceps flexibility, range of motion and foot speed in kicking a football. Journal of Science and Medicine in Sport. 2004; 12: 230-235.

\section{Submitted: 10.11.2018}

\section{Author's information:}

Natalya Yu. Tarabrina - Candidate of Biologics, Moscow Aviation Institute (National Research University), Volokolamskoe highway, House 4, Moscow, A-8o, GSP-3, 125993, e-mail: nata-tarabrina@ mail.ru

Yuriy V. Kraev - Candidate of Psychological Sciences, Moscow Aviation Institute (National Research University), Volokolamskoe highway, House 4, Moscow, A-8o, GSP-3, 125993, e-mail: yury.kraev@mail. $\underline{r u}$ 